\title{
Validation of the BOADICEA model and a 313-variant polygenic risk score for breast cancer risk prediction in a Dutch prospective cohort
}

\author{
Inge M. M. Lakeman, $\mathrm{MD}^{1}$, Mar Rodríguez-Girondo, $\mathrm{PhD}^{2}$, Andrew Lee, $\mathrm{MSc}^{3}$, Rikje Ruiter, $\mathrm{PhD}^{4}$, \\ Bruno H. Stricker, $\mathrm{PhD}^{4}$, Sara R. A. Wijnant, $\mathrm{MD}^{4,5,6}$, Maryam Kavousi, $\mathrm{PhD}^{4}$, \\ Antonis C. Antoniou, $\mathrm{PhD}^{3}$, Marjanka K. Schmidt, $\mathrm{PhD}^{7,8}$, André G. Uitterlinden, $\mathrm{PhD}^{4,9}$, \\ Jeroen van Rooij, $\mathrm{MSc}^{9}$ and Peter Devilee, $\mathrm{PhD} \mathbb{1 0}^{1,10}$
}

Purpose: We evaluated the performance of the recently extended Breast and Ovarian Analysis of Disease Incidence and Carrier Estimation Algorithm (BOADICEA version 5) in a Dutch prospective cohort, using a polygenic risk score (PRS) based on 313 breast cancer $(\mathrm{BC})$-associated variants $\left(\mathrm{PRS}_{313}\right)$ and other, nongenetic risk factors.

Methods: Since 1989, 6522 women without BC aged 45 or older of European descent have been included in the Rotterdam Study. The $\mathrm{PRS}_{313}$ was calculated per $1 \mathrm{SD}$ in controls from the Breast Cancer Association Consortium (BCAC). Cox regression analysis was performed to estimate the association between the $\mathrm{PRS}_{313}$ and incident $\mathrm{BC}$ risk. Cumulative 10 -year risks were calculated with BOADICEA including different sets of variables (age, risk factors and $\left.\mathrm{PRS}_{313}\right)$. C-statistics were used to evaluate discriminative ability.
Results: In total, 320 women developed $\mathrm{BC}$. The $\mathrm{PRS}_{313}$ was significantly associated with $\mathrm{BC}$ (hazard ratio [HR] per SD of 1.56 , $95 \%$ confidence interval [CI] [1.40-1.73]). Using 10-year risk estimates including age and the $\mathrm{PRS}_{313}$, other risk factors improved the discriminatory ability of the BOADICEA model marginally, from a C-statistic of 0.636 to 0.653 .

Conclusions: The effect size of the $\mathrm{PRS}_{313}$ is highly reproducible in the Dutch population. Our results validate the BOADICEA v5 model for $\mathrm{BC}$ risk assessment in the Dutch general population.

Genetics in Medicine (2020) 22:1803-1811; https://doi.org/10.1038/s41436020-0884-4

Keywords: breast cancer; polygenic risk score; prospective cohort; risk assessment

\section{INTRODUCTION}

Breast cancer is the most common cancer among women in Europe. ${ }^{1}$ In the Netherlands, the average lifetime risk for developing invasive breast cancer is $13.6 \%$ for each woman, with the incidence peaking between 60 and 70 years of age. ${ }^{2}$ Mammographic screening has decreased breast cancer mortality at the cost of detecting more disease that otherwise would not have become clinically apparent. ${ }^{3,4}$ Based on the UK guidelines, for every 10,000 women invited for screening at age 50 for the following 20 years, 43 deaths would be prevented, while 129 breast cancers would be overdiagnosed. ${ }^{5}$ Furthermore, breast cancer screening inevitably yields false positives, which can lead to anxiety. ${ }^{6}$ Improvement of this benefit-to-harm ratio could be achieved by targeting women who benefit the most from screening, in particular those in the highest risk categories, while reducing screening for those in the lowest risk categories, potentially reducing overdiagnosis and costs while maintaining a reduced breast cancer death rate and improved quality of life. ${ }^{7}$

Many risk prediction algorithms have been developed to quantify the combined effect of various risk factors to predict the risk of developing breast cancer. ${ }^{8,9}$ The recently extended Breast and Ovarian analysis of Disease Incidence and Carrier Estimation Algorithm (BOADICEA) calculates cumulative risk of developing breast cancer based on family history, mammographic density, and several lifestyle/hormonal and genetic risk factors. ${ }^{10}$ BOADICEA includes the rare high to moderate risk pathogenic variants in breast cancer genes $B R C A 1, B R C A 2, P A L B 2, C H E K 2$, and $A T M$, and a polygenic risk score (PRS) based on 313 breast cancer-associated variants $\left(\mathrm{PRS}_{313}\right)$. In ten prospective studies, this PRS showed an association with breast cancer with an odds ratio (OR) of 1.61 per standard deviation (SD) of the PRS distribution, ${ }^{11}$ and an area under receiver-operator curve of 0.630 . It has

\footnotetext{
${ }^{1}$ Department of Human Genetics, Leiden University Medical Center, Leiden, The Netherlands; ${ }^{2}$ Department of Medical Statistics, Leiden University Medical Center, Leiden, The Netherlands; ${ }^{3}$ Centre for Cancer Genetic Epidemiology, Department of Public Health and Primary Care, University of Cambridge, Cambridge, United Kingdom; ${ }^{4}$ Department of Epidemiology, Erasmus Medical Centre, Rotterdam, The Netherlands; ${ }^{5}$ Department of Respiratory Medicine, Ghent University Hospital, Ghent, Belgium; ${ }^{6}$ Department of

Bioanalysis, Faculty of Pharmaceutical Sciences, Ghent University, Ghent, Belgium; ${ }^{7}$ Department of Clinical Genetics, Leiden University Medical Center, Leiden, The Netherlands; ${ }^{8}$ Division of Molecular Pathology, the Netherlands Cancer Institute, Amsterdam, The Netherlands; ${ }^{9}$ Department of Internal Medicine, Erasmus Medical Centre, Rotterdam, The Netherlands; ${ }^{10}$ Department of Pathology, Leiden University Medical Center, Leiden, The Netherlands. Correspondence: Peter Devilee (p.devilee@lumc.nl)
} 
been shown that the greatest breast cancer risk stratification in the general population and in women with a family history of breast cancer can be obtained by using the combined effects of the PRS and lifestyle/hormonal risk factors in the BOADICEA model. ${ }^{10}$

Currently, breast cancer screening in the Dutch population is age-based. ${ }^{12}$ Women start at age 50 years with biannual mammograms until the age of 75 . Before considering risk-stratified approaches based on BOADICEA, it is important to assess its clinical validity in the Dutch population. In this study we validated the association between the $\mathrm{PRS}_{313}$ and breast cancer in a Dutch prospective cohort, its effect on predicting in situ breast cancer, and explore the discriminative ability of an individualized 10-year breast cancer risk score based on the $\mathrm{PRS}_{313}$ and several known risk factors using the BOADICEA version 5 model. We also assessed how a risk-based approach of population-based screening could have impacted breast cancer detection rates in our study cohort.

\section{Study cohort}

\section{MATERIALS AND METHODS}

The Rotterdam Study (RS) is a prospective population-based cohort study of elderly Dutch individuals living in the Ommoord district of Rotterdam in the Netherlands. ${ }^{13}$ Briefly, in the year 1989, individuals aged 55 or older were recruited into the RS-I cohort, which was extended in 2000 under similar criteria (RS-II cohort) and in 2006 by the inclusion of individuals with an age between 45 and 55 (RS-III cohort). The overall response rate was $72 \%$. In 2008 the Rotterdam Study comprised 14,926 subjects aged 45 years or older, including 8823 women. For our study, we included all 6670 women for whom genotype data were available. Genotyping was not performed for the excluded 2153 women because of a low-quality DNA sample or because they declined blood donation for DNA at study entry.

\section{Ethics statement}

The Rotterdam Study has been approved by the Medical Ethics Committee of the Erasmus Medical Center and by the Dutch Ministry of Health, Welfare, and Sports. All participants provided written informed consent to participate in the study and to have their medical information obtained from treating physicians.

\section{Phenotype data}

Diagnoses of cancer were collected for all individuals up to January 2014 and were based on medical records of general practitioners (including hospital discharge letters) and through linkage with Dutch Hospital Data, Netherlands Comprehensive Cancer Organisation, and histology and cytopathology registries in the region. ${ }^{13}$ In total, 468 women had a breast cancer (invasive or in situ) diagnosis of whom 148 had been diagnosed prior to entry into the Rotterdam Study, and were excluded from further analyses. All participants were interviewed at home at inclusion, underwent extensive examinations every $\sim 5$ years in the Rotterdam Study research facility, and received follow-up questionnaires (Fig. S1), as described elsewhere. ${ }^{13}$ Basic characteristics such as date of birth, vital status, and age at inclusion were known for all participants. For most participants, information on breast cancer risk factors was available (Table S1, total cohort), but family history of breast cancer and mammographic density were lacking. For the analyses, we used only information from the first questionnaire (Fig. S1: RS-I-1, RS-II-1, RS-III-1) at the time of inclusion in the Rotterdam Study for variables that could vary over time, e.g. weight and alcohol use. Age at menopause was only included if menopause occurred before enrollment into the Rotterdam Study (Table S1, subcohort).

\section{Genotype data}

Genotyping was performed with the Illumina $550 \mathrm{~K}$ (RS-I and RS-II cohorts) and $610 \mathrm{~K}$ (RS-III cohort) arrays. ${ }^{13}$ Standard quality control was completed, including selection on European ancestry, and imputation was performed using the Haplotype Reference Consortium (HRC) 1.1 and 1000 G phase 3 reference panels. ${ }^{14,15}$ Of the 313 variants used to calculate the PRS, 28 were directly genotyped by the arrays. Two variants were imputed with a quality below 0.3 and the remaining 283 variants were imputed with an average imputation quality of 0.95 (Table S2).

\section{Polygenic risk score calculation}

The following formula was used to calculate the PRS based on 313 variants:

$P R S_{j}=\sum_{i=1}^{313} n_{i j} \ln \left(O R_{i}\right)$

where $n_{i j}$ is the number of risk alleles $(0,1$, or 2$)$ for variant $i$ carried by individual $j$ and $O R_{i}$ is the per-allele OR for breast cancer associated with variant $i$. The ORs were obtained from the Breast Cancer Association Consortium (BCAC) study ${ }^{11}$ (Table S2). As the estrogen receptor (ER) status of the breast tumors was not available, only the overall breast cancer PRS was calculated. The $\mathrm{PRS}_{313}$ was standardized to the mean in all included women from the Rotterdam Study who did not develop incident breast cancer. To allow for direct comparison of PRS performance between both studies, the SD of the population controls included in the validation set from the BCAC study ${ }^{11}$ was used, which was 0.609 . For the calculations with BOADICEA version 5, the $\mathrm{PRS}_{313}$ was standardized to the mean and SD from the population controls included in the total data set from the BCAC study, ${ }^{11}$ which were -0.424 and 0.603 respectively.

\section{Cumulative risk score calculation}

Cumulative 10-year breast cancer risks were calculated with BOADICEA version $5,{ }^{10}$ starting at the age of inclusion in the 
Rotterdam Study, and using the birth cohort incidence rates in combination with four different sets of variables, i.e., (1) age, (2) age and $\mathrm{PRS}_{313}$, (3) age and risk factors, and (4) age, $\mathrm{PRS}_{313}$, and risk factors. Risk factors included are age at menarche, age at menopause, number of children, age at first live birth, use of oral contraception, use of hormone replacement therapy, body mass index (BMI), height, and alcohol use. For the variables that could vary over time, we used fixed variables. As BOADICEA ignores any risk factors for which the value is missing, ${ }^{10}$ no imputation was performed, and missing variables were kept missing.

Because BOADICEA calculates cumulative breast cancer risks up to age 80,10 -year breast cancer risks were only calculated for 4377 women with an age of inclusion up to 70 years. Women were considered affected if they developed breast cancer (invasive or in situ) within 10 years after inclusion in the Rotterdam Study.

\section{Statistical analyses}

Cumulative incidences were calculated using the Kaplan-Meier method.

\section{Association analyses}

To estimate the association between the $\mathrm{PRS}_{313}$ and breast cancer risk in the Rotterdam Study cohort, Cox regression analyses were performed. Relatedness among individuals of the same family was accounted for by correcting standard errors using a sandwich estimator. All models were adjusted by the age at inclusion in the Rotterdam Study. Incident breast cancer, in situ or invasive, was the event of interest. The time at risk was defined as the time elapsed between the inclusion date and the date of occurrence of the event of interest or right censoring. Right censoring could be due to (1) end of follow-up in January 2014 or (2) death. The proportional hazard assumption for the model was tested. Sensitivity analyses were performed (1) for invasive breast cancer only by censoring the in situ breast cancer cases, (2) for in situ breast cancer only by censoring the invasive breast cancer cases, (3) by censoring at the age of diagnosis of another type of cancer, and (4) by stratifying on Rotterdam Study cohort. To define the association between the $\mathrm{PRS}_{313}$ and other tumors than breast cancer, similar Cox regression analysis was performed by censoring the breast cancer cases if they did not develop another tumor before the breast cancer diagnosis.

To investigate if the linearity assumption for the effect of $\mathrm{PRS}_{313}$ holds, we ran the model considering the categorical covariate given by the percentile groups of the $\mathrm{PRS}_{313}(0-10 \%$, $10-20 \%$, 20-40\%, reference $40-60 \%, 60-80 \%$, 80-90\%, $90-100 \%)$ based on the distribution in the unaffected women in this cohort. The discrimination ability of the $\mathrm{PRS}_{313}$ in our sample was evaluated using the C-statistic, ${ }^{16}$ by groups based on quantiles of the age of inclusion in the Rotterdam Study (i.e. age $<60,60-70$, and $\geq 70$ years). Differences in the Cstatistics were tested by computing bootstrap CIs for the differences among groups.

\section{Age-varying effect}

The possible time-varying association of the $\mathrm{PRS}_{313}$ with breast cancer was investigated using age as time scale and considering three age-dependent coefficients in the Cox model, corresponding to three different age intervals: (1) younger than 50 years, (2) between 50 and 75 years old, and (3) above 75 years old. These cut-offs were chosen based on their clinical relevance since women between 50 and 75 years are eligible for population screening according to the Dutch guideline. ${ }^{12}$

\section{Clinical validity of BOADICEA v5}

To validate the BOADICEA 10-year cumulative risk scores, model calibration and discrimination ability in our sample were assessed. Calibration was investigated by comparing overall observed versus expected cumulative risks and by visually inspecting the calibration plots based on risk deciles. Because of the presence of right censoring, empirical risks at 10 years were estimated using the Kaplan-Meier method. As in the association analyses, discrimination was evaluated using C-statistics.

Statistical significance was defined as a two-sided $p$ value of $<0.05$. All analyses were performed with $\mathrm{R}$ version $3.5 .3 .{ }^{17}$

\section{RESULTS}

We included 6522 women in the main analyses with an average age at study entry of 66 years. Of these, 320 developed either invasive or in situ breast cancer during follow-up and 744 developed another type of tumor; the overlap between these two groups was 16, all of whom developed another type of tumor first (Table S3). The median follow-up calculated with the reverse Kaplan-Meier method was 12.40 years, with a minimum and maximum follow-up of 0.03 and 24.43 years. Cohort characteristics are shown in Table S1. The average $\mathrm{PRS}_{313}$ in groups of affected (i.e. invasive, in situ, and a second breast tumor) and unaffected women (including women who developed another tumor than breast cancer) are shown in Fig. S2 and Table S4.

\section{Breast cancer cumulative incidence}

The cumulative incidence of breast cancer in the total cohort was on average $4.2 \%, 95 \%$ CI [3.7\%-4.8\%] and 7.3\%, 95\% CI [6.4\%-8.2\%] 10 and 20 years after inclusion respectively. Stratified by quintiles of the $\mathrm{PRS}_{313}$, after 20 years of followup, the incidence in the highest quintile was $10.8 \%, 95 \% \mathrm{CI}$ [8.5\%-13.1\%] and 4.4\%, 95\% CI [2.8\%-6.0\%] for the lowest quintile (Fig. S3).

\section{Association analyses}

A significant association was found between the $\mathrm{PRS}_{313}$ and incident breast cancer with an $\mathrm{HR}$ per SD of $1.56,95 \% \mathrm{CI}$ [1.40-1.74], $p=2.47 \times 10^{-15}$ (Table 1). There was no evidence of violation of the proportional hazard assumption ( $p$ value $=0.716)$, indicating that the $\mathrm{HR}$ remained constant over time. The discriminative ability of the $\mathrm{PRS}_{313}$, as measured by the C-statistic, was 0.632 , 95\% CI [0.58-0.69], 
Table 1 Results of the association analyses between breast cancer and the PRS 313 .

\begin{tabular}{|c|c|c|c|c|c|c|c|}
\hline & $n$ Included & $n$ Events & HR & $95 \% \mathrm{Cl}$ & $p$ value & C-statistic ${ }^{c}$ & $95 \% \mathrm{Cl}$ \\
\hline Main analyses & 6522 & 320 & 1.56 & $1.40-1.74$ & $2.47 \times 10^{-15}$ & & \\
\hline \multicolumn{8}{|c|}{ Age category for discriminative ability of the PRS } \\
\hline $60-70$ & 2174 & 128 & & & & 0.673 & $0.61-0.73$ \\
\hline$\geq 70$ & 2173 & 88 & & & & 0.562 & $0.48-0.62$ \\
\hline \multicolumn{8}{|l|}{ Sensitivity analyses } \\
\hline In situ BC only & 6522 & 34 & 1.43 & $1.01-2.01$ & 0.042 & & \\
\hline Censored at other tumor & $6402^{b}$ & 298 & 1.54 & $1.37-1.73$ & $1.88 \times 10^{-13}$ & & \\
\hline Stratified by RS cohort & 6522 & 320 & 1.56 & $1.40-1.75$ & $1.92 \times 10^{-15}$ & & \\
\hline \multicolumn{8}{|l|}{ Percentage of the PRS } \\
\hline $0-10 \%$ & 637 & 17 & 0.59 & $0.34-1.01$ & 0.053 & & \\
\hline $80-90 \%$ & 656 & 36 & 1.28 & $0.84-1.94$ & 0.251 & & \\
\hline $90-100 \%$ & 687 & 67 & 2.37 & $1.66-3.37$ & $1.73 \times 10^{-06}$ & & \\
\hline \multicolumn{8}{|c|}{ Age category for time-varying analyses } \\
\hline$<50$ & 224 & 2 & 2.74 & $1.72-4.37$ & $2.23 \times 10^{-05}$ & & \\
\hline $50-75$ & 5104 & 197 & 1.74 & $1.52-2.00$ & $2.21 \times 10^{-15}$ & & \\
\hline$>75$ & 4032 & 121 & 1.29 & $1.08-1.55$ & 0.005 & & \\
\hline
\end{tabular}

$B C$ breast cancer, $C I$ confidence interval, $H R$ hazard ratio, $P R S$ polygenic risk score, $R S$ Rotterdam Study.

${ }^{a} 4$ women developed an invasive breast tumor after development of an in situ breast tumor.

b120 women were excluded from analyses because they developed another tumor before inclusion in the Rotterdam Study.

${ }^{\mathrm{C}}$ The corresponding differences in C-statistic were for women with inclusion age 60-70 versus age $<60$ : $0.041,95 \% \mathrm{Cl}$ [-0.05-0.12]; for women with inclusion age $60-70$ versus age $\geq 70: 0.111,95 \% \mathrm{Cl}[0.02-0.21]$; for women with inclusion age $<60$ versus age $\geq 70: 0.070,95 \% \mathrm{Cl}[-0.01-0.18]$.

$0.673,95 \%$ CI [0.61-0.73], and 0.562, 95\% CI [0.48-0.62] for women included before age 60 , between age 60 and 70 , and above age 70 respectively (Table 1 ).

Sensitivity analyses for (1) invasive breast cancer only, (2) censoring at another tumor if applicable, or (3) stratifying by the Rotterdam Study subcohort all showed similar results (Table 1). Notably, in situ breast cancer also showed a statistically significant association with the $\mathrm{PRS}_{313}, \mathrm{HR}$ per $\mathrm{SD}=1.43,95 \% \mathrm{CI}[1.01-2.01], p=0.042$.

Association analyses for breast cancer and percentiles of the $\mathrm{PRS}_{313}$ showed that the HR estimates were in line with the HR predicted when a continuous $\mathrm{PRS}_{313}$ is assumed, under a loglinear model (Fig. 1, Table 1).

During follow-up, 744 women developed a tumor other than breast cancer without evidence for association with the $\mathrm{PRS}_{313}(\mathrm{HR}$ per $\mathrm{SD}=1.05,95 \%$ CI $[0.98-1.12], p$ value $=$ 0.195).

\section{Age-varying effect}

Extension of the Cox model allowing for age-dependent regression coefficients showed that the performance of the $\mathrm{PRS}_{313}$ decreased with increasing inclusion age, with the HRs per SD declining from $2.74,95 \%$ CI [1.72-4.37] for women included before age 50 , to $1.74,95 \%$ CI [1.52-2.00] for women included between 50 and $75\left(p_{\text {diff }}=0.066\right)$. The HR for women included after age 75 was 1.29 , 95\% CI [1.08-1.55], and the $p$ value of the difference with respect to the youngest group was 0.003 (Table 1 ).

\section{Clinical validity of BOADICEA V5}

For these analyses, we selected 4377 women with an age of inclusion under 70 years. Of these, 163 developed breast cancer within 10 years after inclusion (142 invasive). The median follow-up in this subcohort was 10 years (range 0.03-10 years), and the cumulative incidence of breast cancer was $4.4 \%$ (95\% CI [3.7-5.1\%]). The distributions of 10 -year cumulative risk scores under different models are shown in Figs. S4 and S5. Irrespective of the variables included, BOADICEA underestimated the observed risk of $4.4 \%$ (Table 2). Accordingly, while using age and $\mathrm{PRS}_{313}$ seems to result in the best calibration (Fig. S4C), it underestimated the observed risks in the higher risk categories. The highest discriminative ability was found for the model with age, $\mathrm{PRS}_{313}$ and all available risk factors $(0.653,95 \% \mathrm{CI}$ [0.60-0.70]), henceforth the "full" model. The $\mathrm{PRS}_{313}$ was the strongest factor contributing to discrimination, relative to age and other risk factors (Table 2).

Using the full model and a threshold of 2.5\% 10-year breast cancer risk, which approximates the risk of women entering the age-based population screening program in the 


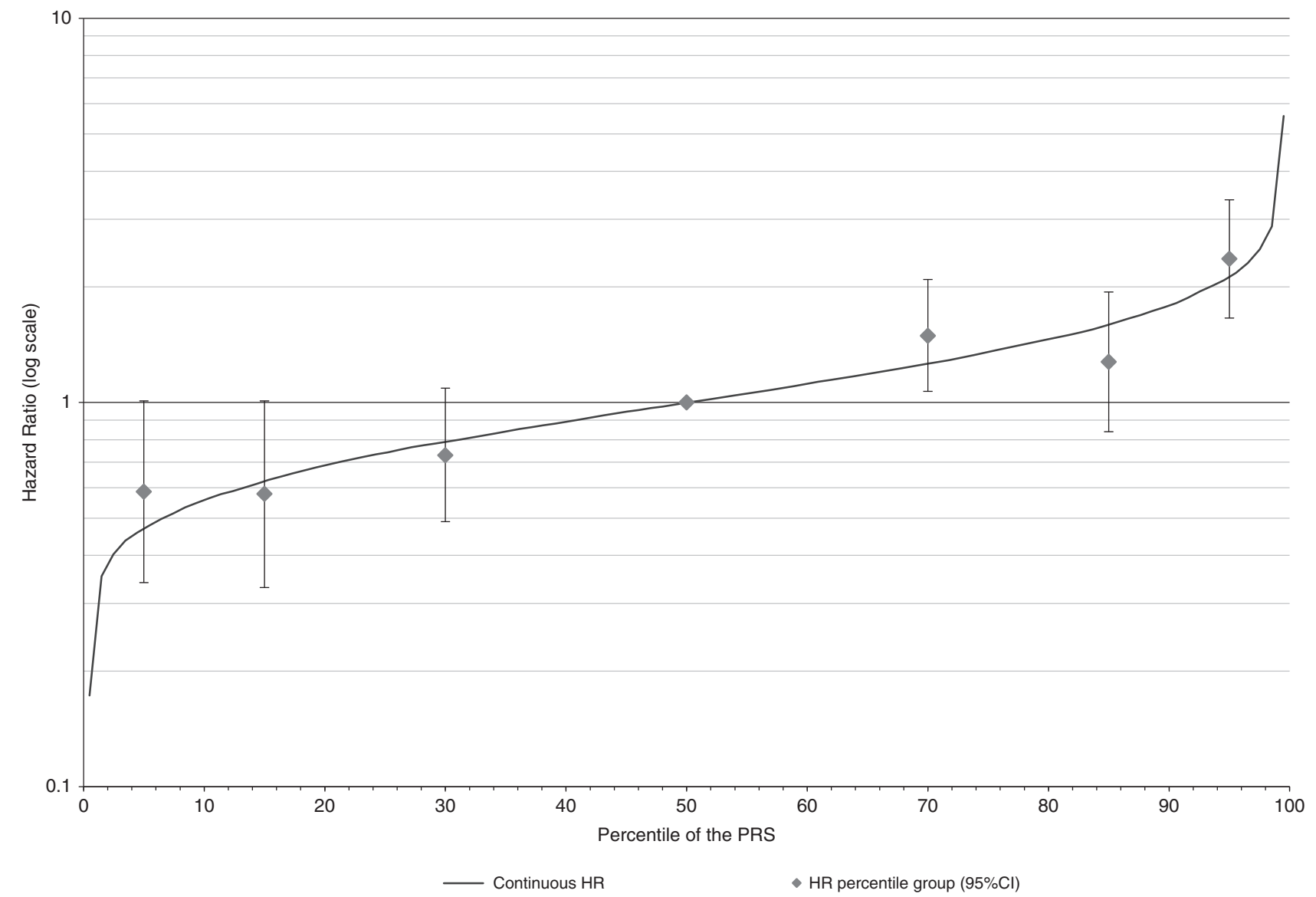

Fig. 1 Association with the $\mathrm{PRS}_{313}$ and breast cancer risk. Plot of the HR for the association between the $\mathrm{PRS}_{313}$ and breast cancer risk based on PRS 313 percentiles. The $\mathrm{PRS}_{313}$ percentile groups are $0-10 \%, 10-20 \%, 20-40 \%, 40-60 \%$ (reference), $60-80 \%, 80-90 \%, 90-100 \%$ based on the distribution in unaffected women. The numbers and corresponding effect sizes are shown in Table 1 . The solid line represents the continuous distribution based on the per SD effect size of the $\mathrm{PRS}_{313}$. Cl confidence interval, $H R$ hazard ratio, $P R S$ polygenic risk score.

Table 2 Range and discriminative ability of the cumulative 10-year breast cancer risk scores calculated with BOADICEA.

Variables included

Age

Age, PRS $_{313}$

Age, risk factors, $\mathrm{PRS}_{313}$
Mean \% (range)

Unaffected women

C-statistic

BC cases $^{\mathrm{a}}$

$\begin{array}{lll}2.9(2.2-3.6) & 0.531 & 0.50-0.58 \\ 2.6(1.4-4.3) & 0.558 & 0.52-0.60 \\ 3.8(1.2-8.3) & 0.636 & 0.59-0.68 \\ 3.3(0.9-10.5) & 0.653 & 0.60-0.70\end{array}$

$B C$ breast cancer, $B O A D I C E A$ Breast and Ovarian Analysis of Disease Incidence and Carrier Estimation Algorithm, $C$ confidence interval, PRS polygenic risk score. Women who developed $B C$ within 10 years of follow-up.

Netherlands, 101 cases (62\% of incident cases) occurred in a screening group of 1956 women (45\% of total) and 62 breast cancers occurred in 2421 women who would not be screened (Fig. 2; Table 3). Using the $\mathrm{PRS}_{313}$ and age only, 130 cases ( $80 \%$ of incident cases) occurred in a screening group of 2863 women $(65 \%$ of total); in 1481 women who would not be screened, 33 breast cancers occurred. In Fig. S6 the percentages of incident breast cancer cases and unaffected women are shown for different category thresholds. For both models, the invasive cancers in the group selected for screening were more likely to be of lower grade compared with the cancers in the nonscreened group (Table 3). The reverse effect was found for in situ cancers.

\section{DISCUSSION}

Many risk factors for breast cancer, both genetic and nongenetic, have been identified in the past decades. ${ }^{18,19}$ Increasingly, these are being integrated into computational models that allow personalized breast cancer risk assessment, which has potential application beyond current practice of 

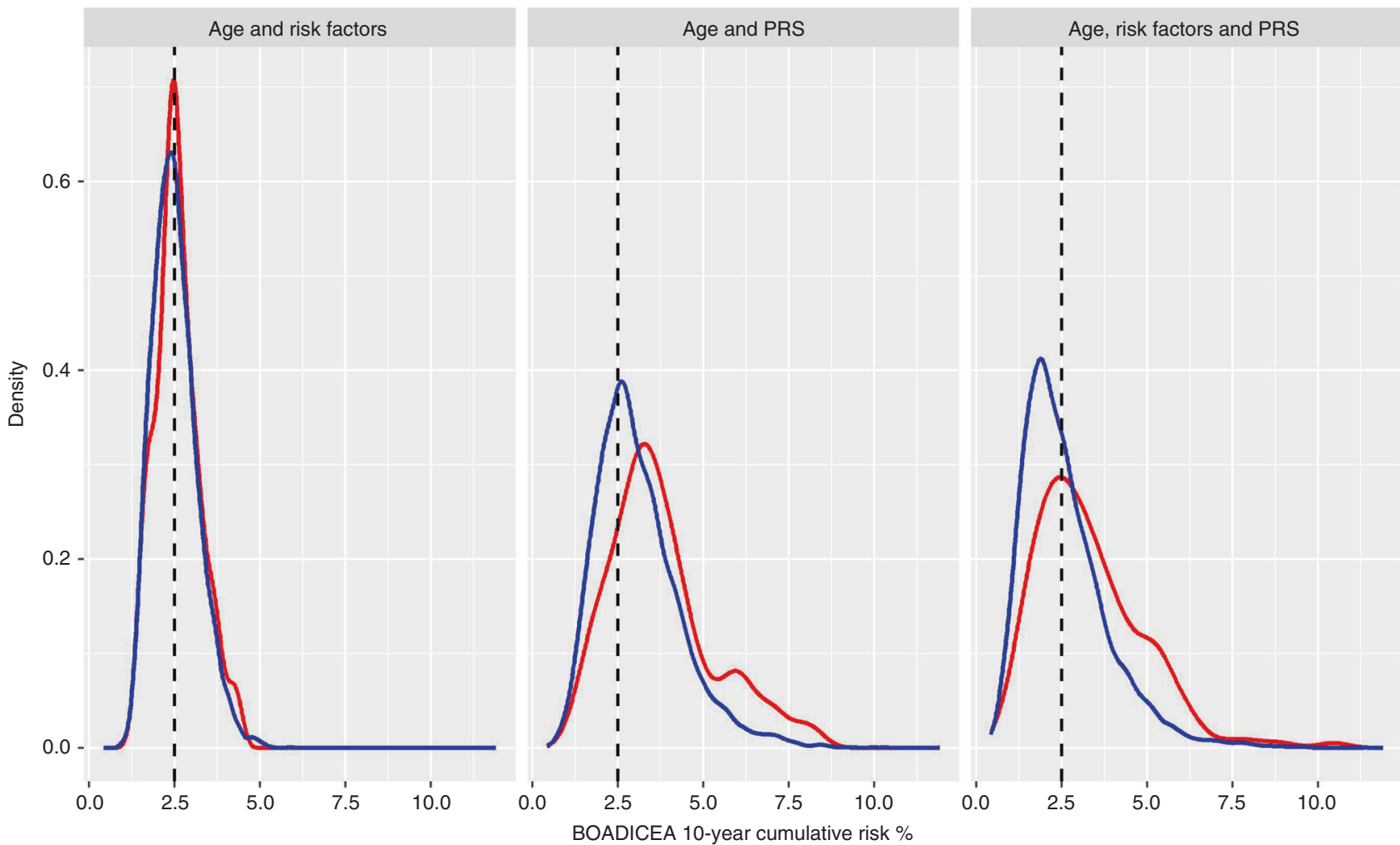

- Incident BC cases, $\mathrm{N}=163$ Unaffected women, $\mathrm{N}=4214$

Fig. 2 Cumulative 10-year breast cancer risk distribution predicted by BOADICEA. Density plots of the cumulative 10-year risk calculated by BOADICEA for unaffected women and incident breast cancer cases. Including age and risk factors (left), including age and the PRS ${ }_{313}$ (middle), and the full model including age, risk factors and the $\mathrm{PRS}_{313}$. The dashed line shows the threshold of a 10 -year risk of $2.5 \%$. BOADICEA Breast and Ovarian Analysis of Disease Incidence and Carrier Estimation Algorithm, PRS polygenic risk score.

Table 3 Numbers and percentages of women per 10-year risk category.

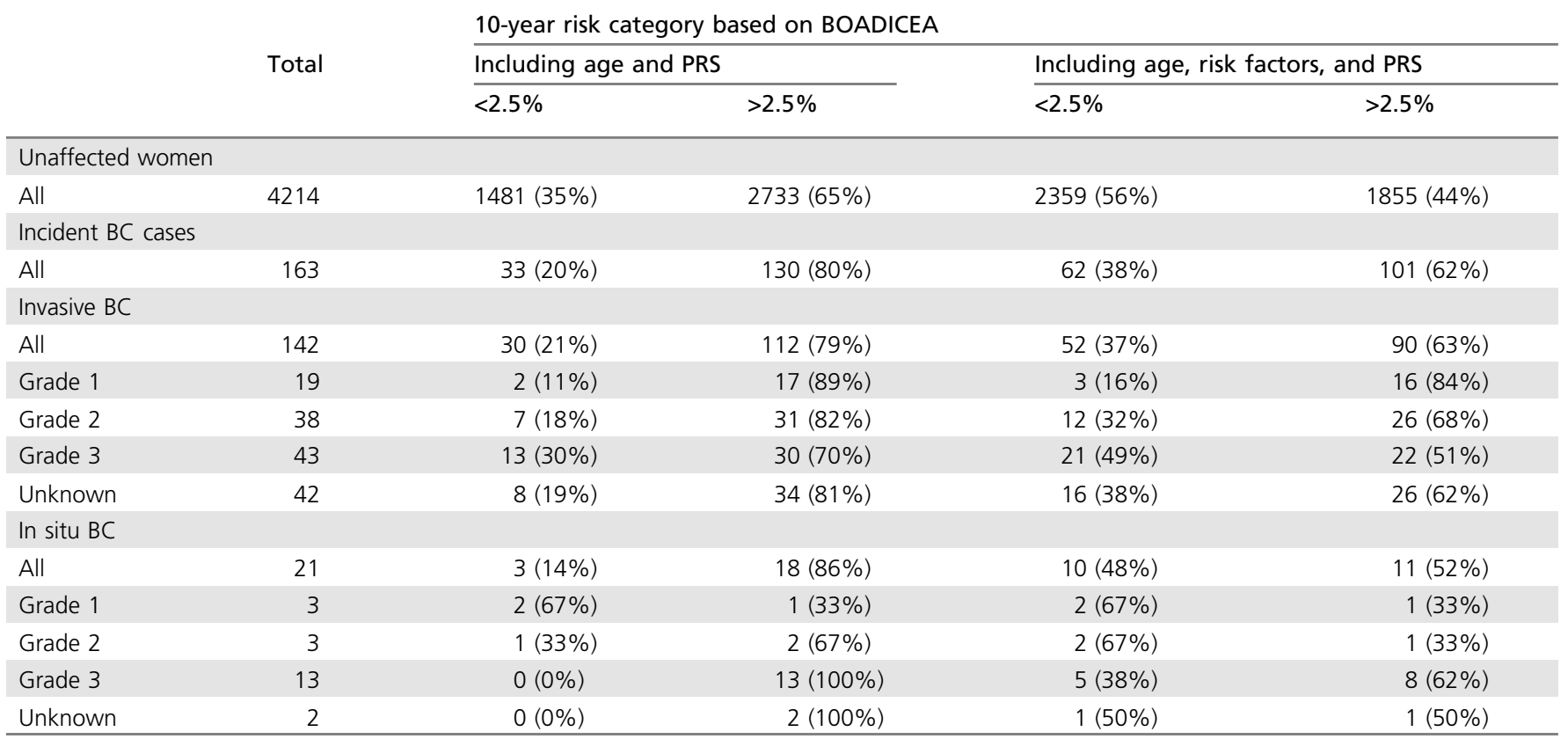

$B C$ breast cancer, BOADICEA Breast and Ovarian Analysis of Disease Incidence and Carrier Estimation Algorithm, PRS polygenic risk score. 
genetic testing in family cancer clinics. ${ }^{8,9,20}$ The BOADICEA algorithm is among the most comprehensive risk models presently available for breast cancer risk assessment. ${ }^{10}$ Here, we validated the most recent version of this model in a large prospective population-based Dutch cohort of women above 45 years, which has not been part of the previously published BCAC study. ${ }^{11}$ Unsurprisingly, the best discrimination was achieved after inclusion of all available risk factors, with the largest contribution deriving from the $\mathrm{PRS}_{313}$. The $\mathrm{PRS}_{313}$ was significantly associated with breast cancer, with a similar effect size as in other prospective series of different geographic origin, ${ }^{11}$ demonstrating its robustness and potential application to the Dutch population.

The PRS ${ }_{313}$ improved the discriminatory ability from 0.531 to 0.636 , compared with a model using age only, which could only be marginally improved further (to 0.653 ) by adding lifestyle, reproductive factors, and anthropometric data. This is in line with previous research, showing that the variance explained by the risk factors is modest compared with the $\mathrm{PRS}_{313}$ risk stratification. ${ }^{10,21}$ Results of the calibration showed that BOADICEA underestimated the observed risks, especially in the higher categories of risk. One possible explanation is that BOADICEA v5 uses the population breast cancer incidences of the United Kingdom as baseline risk, which are slightly lower than those in the Netherlands. ${ }^{1}$ But more importantly, data on family history, mammographic density, and rare high-risk variants in BRCA1 and $B R C A 2$ were lacking in our cohort. In another prospective validation study of a previous version of BOADICEA in two cohorts of women from Australia, Canada, and the United States, information on family history and BRCA1/2 carrier status, but not the $\mathrm{PRS}_{313}$, was available, and here, BOADICEA overestimated 10-year cumulative risks in the highest risk quantile. ${ }^{9}$ Possibly, the missing data on family history and $B R C A 1 / 2$ status in the Rotterdam Study were in fact more prevalent than modeled by BOADICEA. Our calibration results indicate that for proper use in the general population, information on family history may be important.

We illustrated the potential impact of the model in detecting breast cancer in a population screening setting in which women would participate based on their individual risk. In this illustration, the $\mathrm{PRS}_{313}$ alone would have detected more cases than the full BOADICEA model, but would also have identified a larger screening group. Apparently, women in the Rotterdam Study have on average fewer nongenetic risk factors compared with the total population, which on average slightly modifies their risk in a downward direction. The PROCAS study used the Tyrer-Cuzick model with mammographic density and risk factors, combined with a PRS based on 18 single-nucleotide polymorphisms (SNPs); ${ }^{22}$ they found $82 \%$ of the cases to occur in $68 \%$ of women with a 10 -year breast cancer risk above $2 \%$, i.e., very similar to what we found with the $\mathrm{PRS}_{313}$ alone.

Remarkably, we found the proportion of low grade invasive tumors to be higher in those with a 10 -year risk $>2.5 \%$, compared with those with lower risks. Screen-detected invasive cancers are more likely of lower grade and stage. ${ }^{23}$ Our cohort data did not include information on whether incident breast cancers were screen-detected or not, hence we cannot exclude that high-risk women disproportionally selfselected for mammographic screening, which could explain this bias. In contrast, for the in situ carcinomas, more high grade tumors were found in the $>2.5 \% 10$-year risk group compared with those with lower risks. Histological grade of ductal carcinoma in situ (DCIS) has been suggested to be one of six factors associated with subsequent development of invasive disease, ${ }^{24}$ albeit not very strongly so. It remains possible that the $\mathrm{PRS}_{313}$ is more strongly associated with low grade invasive breast cancer than with higher grades, as observed for some individual variants, ${ }^{25,26}$ and inversely so for DCIS. It will be important to replicate this in larger studies to inform the evaluation of the cost-effectiveness of a risk-based versus age-based entry of the population screening. ${ }^{7}$

Although PRS development studies have included only invasive breast cancer, ${ }^{11,27}$ in our cohort the $\mathrm{PRS}_{313}$ is associated with in situ breast cancer as well, with a nonsignificantly lower effect size than for invasive breast cancer. This corresponds well with a previously reported association of an 18-SNP-based PRS $^{22}$ and with previous results showing that the association of 51 of the 76 investigated breast cancer loci with DCIS is in the same direction as for invasive breast cancer. ${ }^{28}$ Although BOADICEA is presented as a model that predicts invasive breast cancer, ${ }^{10}$ these results suggest it might also predict in situ breast cancer. Larger studies are needed to confirm this and provide more accurate risk estimates, specifically in the setting of population screening programs.

As in previous studies, ${ }^{11,27}$ we found that the effect size of the PRS for breast cancer declined with increasing age. While this is not yet modeled in BOADICEA, this could be important to consider for women under the age of 50 who are at this moment not eligible for population breast cancer screening in the Netherlands, because our results suggest that using the overall HR would be underestimating risk in this age group.

In the Rotterdam Study, malignancies other than breast cancer are also recorded. We found no evidence for association of the $\mathrm{PRS}_{313}$ with these cancers, suggesting it specifically predicts breast cancer. Another prospective study also reported no association between other types of cancer and a sum of breast cancer risk alleles at 72 loci. ${ }^{29}$ Because we only analyzed all other tumors combined, we cannot exclude that the $\mathrm{PRS}_{313}$ has an association with one specific type of other cancer.

A strength of our study is the prospective population-based study design, including all women in a specified locale near Rotterdam. Because of the high response rate $(>70 \%)$ it is a good representation of the Dutch population in that age category. ${ }^{13}$ Furthermore, for a large group of women, there is extensive follow-up of up to 25 years.

Besides that information on mammographic density and family history was lacking, another limitation of our study is 
the unknown ER status of the breast tumors, precluding the analysis of ER-positive and ER-negative disease separately. Furthermore, to evaluate the introduction of risk-based entry into population screening, establishing the detection rate of breast cancers below the age of 50 would have been relevant, which was not possible in our older cohort of women. Finally, we excluded nearly $25 \%$ of all women in the Rotterdam Study because no genotyping data were available. Declining blood donation for DNA extraction did not lead to differences in the basic characteristics between the genotyped and nongenotyped groups. Therefore, if a selection bias was present, we believe this bias would be small.

In summary, the $\mathrm{PRS}_{313}$ replicates robustly in the Dutch population and the discriminative power of the BOADICEA model seems appropriate for implementation into breast cancer prevention programs, such as those currently ongoing in cancer family clinics in many countries worldwide. However, application to the general population would require recalibration of BOADICEA to address underestimation in the higher risk categories. Although the Rotterdam Study design precluded analysis of breast cancer-specific mortality, our evaluation of clinical validity provides first insights into how a risk-based entry could impact the efficacy of the breast cancer population screening program in the Netherlands.

\section{SUPPLEMENTARY INFORMATION}

The online version of this article (https://doi.org/10.1038/s41436020-0884-4) contains supplementary material, which is available to authorized users.

\section{ACKNOWLEDGEMENTS}

This work was supported by the Dutch Cancer Society (KWF), grant UL2014-7473. The Rotterdam Study is funded by Erasmus Medical Center and Erasmus University, Rotterdam; Netherlands Organization for the Health Research and Development (ZonMw); the Research Institute for Diseases in the Elderly (RIDE); the Ministry of Education, Culture and Science; the Ministry for Health, Welfare, and Sports; the European Commission (DG XII); and the Municipality of Rotterdam. The authors are grateful to the study participants, the staff from the Rotterdam Study and the participating general practitioners and pharmacists. The generation and management of genome-wide association study (GWAS) genotype data for the Rotterdam Study (RS-I, RS-II, RS-III) was executed by the Human Genotyping Facility of the Genetic Laboratory of the Department of Internal Medicine, Erasmus MC, Rotterdam, the Netherlands. The GWAS data sets are supported by the Netherlands Organisation of Scientific Research NWO Investments (175.010.2005.011, 911-03-012), the Genetic Laboratory of the Department of Internal Medicine, Erasmus MC, the Research Institute for Diseases in the Elderly (014-93-015; RIDE2), the Netherlands Genomics Initiative (NGI)/ Netherlands Organisation for Scientific Research (NWO) Netherlands Consortium for Healthy Aging (NCHA), project number 050-060-810. We thank Pascal Arp, Mila Jhamai, Marijn Verkerk, Lizbeth Herrera, Marjolein Peters, and Carolina Medina-Gomez for their help in creating the GWAS database, and Karol Estrada,
Yurii Aulchenko, and Carolina Medina-Gomez, for the creation and analysis of imputed data.

\section{DISCLOSURE}

The authors declare no conflicts of interest.

Publisher's note Springer Nature remains neutral with regard to jurisdictional claims in published maps and institutional affiliations.

\section{REFERENCES}

1. Ferlay J, Colombet M, Soerjomataram I, et al. Cancer incidence and mortality patterns in Europe: estimates for 40 countries and 25 major cancers in 2018. Eur J Cancer. 2018;103:356-387.

2. van der Waal $D$, Verbeek $A L$, den Heeten GJ, Ripping TM, Tjan-Heijnen VC, Broeders MJ. Breast cancer diagnosis and death in the Netherlands: a changing burden. Eur J Public Health. 2015;25:320-324.

3. Marmot MG, Altman DG, Cameron DA, Dewar JA, Thompson SG, Wilcox $M$. The benefits and harms of breast cancer screening: an independent review. Br J Cancer. 2013;108:2205-2240.

4. Ripping TM, Verbeek AL, Fracheboud J, de Koning HJ, van Ravesteyn NT, Broeders MJ. Overdiagnosis by mammographic screening for breast cancer studied in birth cohorts in The Netherlands. Int J Cancer. 2015;137:921-929.

5. Marmot MG, Altman DG, Cameron DA, Dewar JA, Thompson SG, Wilcox M. The benefits and harms of breast cancer screening: an independent review. Lancet. 2012;380:1778-1786.

6. Hubbard RA, Kerlikowske K, Flowers Cl, Yankaskas BC, Zhu W, Miglioretti DL. Cumulative probability of false-positive recall or biopsy recommendation after 10 years of screening mammography: a cohort study. Ann Intern Med. 2011;155:481-492.

7. Pashayan N, Morris S, Gilbert FJ, Pharoah PDP. Cost-effectiveness and benefit-to-harm ratio of risk-Stratified screening for breast cancer: a lifetable model. JAMA Oncol. 2018;4:1504-1510.

8. Cintolo-Gonzalez JA, Braun D, Blackford AL, et al. Breast cancer risk models: a comprehensive overview of existing models, validation, and clinical applications. Breast Cancer Res Treat. 2017;164:263-284.

9. Terry MB, Liao Y, Whittemore AS, et al. 10-year performance of four models of breast cancer risk: a validation study. Lancet Oncol. 2019:20:504-517

10. Lee A, Mavaddat N, Wilcox AN, et al. BOADICEA: a comprehensive breast cancer risk prediction model incorporating genetic and nongenetic risk factors. Genet Med. 2019:21:1708-1718.

11. Mavaddat N, Michailidou K, Dennis J, et al. Polygenic risk scores for prediction of breast cancer and breast cancer subtypes. Am J Hum Genet. 2019;104:21-34.

12. IKNL. Netherlands Comprehensive Cancer Organisation: Oncoline Mammacarcinoom. 2019. www.oncoline.nl/richtlijn/item/index.php? pagina=/richtlijn/item/pagina.php\&richtlijn_id=885. Accessed December 2019.

13. Ikram MA, Brusselle G, Ghanbari M, et al. Objectives, design and main findings until 2020 from the Rotterdam Study. Eur J Epidemiol. 2020:35:483-517

14. McCarthy S, Das S, Kretzschmar W, et al. A reference panel of 64,976 haplotypes for genotype imputation. Nat Genet. 2016;48:1279-1283.

15. Auton A, Brooks LD, Durbin RM, et al. A global reference for human genetic variation. Nature. 2015;526:68-74.

16. Uno H, Cai T, Pencina MJ, D'Agostino RB, Wei $\amalg$. On the C-statistics for evaluating overall adequacy of risk prediction procedures with censored survival data. Stat Med. 2011;30:1105-1117.

17. R Core Team. R: a language and environment for statistical computing Vienna, Austria: R Foundation for Statistical Computing; 2019.

18. Rojas K, Stuckey A. Breast cancer epidemiology and risk factors. Clin Obstet Gynecol. 2016:59:651-672.

19. Lakeman IMM, Schmidt MK, van Asperen CJ, Devilee P. Breast cancer susceptibility-towards individualised risk prediction. Curr Genet Med Rep. 2019;7:124-135.

20. Turnbull C, Sud A, Houlston RS. Cancer genetics, precision prevention and a call to action. Nat Genet. 2018;50:1212-1218.

21. Maas $P$, Barrdahl M, Joshi AD, et al. Breast cancer risk from modifiable and nonmodifiable risk factors among white women in the United States. JAMA Oncol. 2016;2:1295-1302. 
22. van Veen EM, Brentnall $A R$, Byers $H$, et al. Use of single-nucleotide polymorphisms and mammographic density plus classic risk factors for breast cancer risk prediction. JAMA Oncol. 2018;4:476-482.

23. Autier P, Boniol M, Koechlin A, Pizot C, Boniol M. Effectiveness of and overdiagnosis from mammography screening in the Netherlands: population based study. BMJ. 2017;359:j5224.

24. Visser LL, Groen EJ, van Leeuwen FE, Lips EH, Schmidt MK, Wesseling J. Predictors of an invasive breast cancer recurrence after DCIS: a systematic review and meta-analyses. Cancer Epidemiol Biomarkers Prev. 2019;28:835-845

25. Garcia-Closas M, Hall P, Nevanlinna $H$, et al. Heterogeneity of breast cancer associations with five susceptibility loci by clinical and pathological characteristics. PLoS Genet. 2008;4:e1000054.

26. Purrington KS, Slettedahl S, Bolla MK, et al. Genetic variation in mitotic regulatory pathway genes is associated with breast tumor grade. Hum Mol Genet. 2014;23:6034-6046.

27. Mavaddat N, Pharoah PD, Michailidou K, et al. Prediction of breast cancer risk based on profiling with common genetic variants. J Natl Cancer Inst. 2015;107:djv036

28. Petridis C, Brook MN, Shah V, et al. Genetic predisposition to ductal carcinoma in situ of the breast. Breast Cancer Res. 2016;18:22.
29. Naslund-Koch C, Nordestgaard BG, Bojesen SE. Common breast cancer risk alleles and risk assessment: a study on 35,441 individuals from the Danish general population. Ann Oncol. 2017; 28:175-181.

(i) Open Access This article is licensed under a Creative Commons Attribution 4.0 International License, which permits use, sharing, adaptation, distribution and reproduction in any medium or format, as long as you give appropriate credit to the original author(s) and the source, provide a link to the Creative Commons license, and indicate if changes were made. The images or other third party material in this article are included in the article's Creative Commons license, unless indicated otherwise in a credit line to the material. If material is not included in the article's Creative Commons license and your intended use is not permitted by statutory regulation or exceeds the permitted use, you will need to obtain permission directly from the copyright holder. To view a copy of this license, visit http://creativecommons.org/licenses/ by/4.0/.

(c) The Author(s) 2020 